\title{
Analyse de l'activité des enfants avec une interface tangible et narrative (Lunii - La fabrique à histoires) : un instrument au service de la créativité ?
}

\author{
Analysis of children's activity with a tangible and narrative interface \\ (Lunii - La fabrique à histoires): an instrument for creativity?
}

\author{
Laura Anastasio Forcisi ${ }^{1}$, Françoise Decortis ${ }^{1}$ \\ ${ }^{1}$ Laboratoire Paragraphe, Equipe C3U, Université Paris 8, France, forcisilaura@gmail.com, fdecortis@gmail.com
}

\begin{abstract}
RÉSUMÉ. L'objectif de cet article est d'analyser l'activité des enfants utilisant un dispositif numérique et tangible permettant de créer des histoires et de les écouter (Lunii - La fabrique à histoires). Cette étude, basée sur dix enfants, repose sur la démarche ergonomique visant à étudier un artefact en situation. Les données recueillies sont traitées sous l'angle de l'approche instrumentale de Rabardel afin de poser les schèmes et genèses présents lors de l'usage de Lunii. Nous mobilisons de manière complémentaire le cycle de l'activité créatrice de l'imagination de Vygotski en vue de répondre à la question suivante : quelle est l'influence de cet artefact narratif et connecté sur le développement de la créativité ?

ABSTRACT. We propose in this article to analyse children's activity during the use of a digital and tangible artifact destined to create and listen stories (Lunii - La fabrique à histoires). Our study, based on ten childrens, builds on ergonomics process study an artefact in use. Collected data are analysed through Rabardel's instrumental theory to set up schemes and genesis present during Lunii's use. We mobilize complementarily Vygotski's theory of creative imagination to answer to this question: what is the influence of this narrative and connected artefact on the development of the creativity?

MOTS-CLÉS. Analyse de l'activité, Enfant, Théorie instrumentale, Créativité, Interface tangible, Récit.

KEYWORDS. Analysis of activity, Children, Instrumental theory, Creativity, Tangible interface, Narrative.
\end{abstract}

\section{Introduction}

Dans cet article, nous nous s'intéressons à des outils qui pourraient potentiellement soutenir le développement de la créativité chez l'enfant. Nous regardons quelles sont les propriétes de différentes interfaces de composition-création narrative et leurs effets sur l'activité. Dans l'étude réalisée ici, auprès de dix enfants (âgés de quatre à dix ans), nous cherchons à analyser les dimensions de leur activité lors de l'utilisation d'un objet connecté : Lunii - La fabrique à histoires. Cet artefact, conçu à destination des enfants, est une interface tangible permettant de fabriquer et d'écouter une quarantaine d'histoires. Lunii est utilisé en situation, intégré dans le quotidien et l'environnement familial. On accorde une place centrale au contexte qui influence indubitablement l'usage de tout artefact : l'action est orientée vers un objectif et dépend d'une multitude de caractéristiques propres à la situation (environnementales, sociales, matérielles, etc.) [SUC 87] mais aussi propres au sujet. Il est important d'observer les objets technologiques en situation naturelle et non dans un cadre simulé, en s'inscrivant dans une démarche longitudinale et qualitative auprès de quelques sujets afin de comprendre en profondeur leurs motivations intrinsèques.

L'activité des enfants est analysée selon l'approche instrumentale [RAB 95] puisqu'elle permet d'analyser les moyens de l'activité dans une situation singulière, en restant au plus près du point de vue du sujet. La mise en perspective de l'activité instrumentée avec cette approche peut conduire à mettre en évidence les dimensions de l'activité empêchées, ou contrariées par l'artefact ainsi que les dimensions de l'activité enrichies par Lunii. Nous tentons ici d'analyser les transformations de l'activité provoquées par l'introduction d'un artefact technologique, en adoptant une perspective anthropocentrée sur la technique et en s'inscrivant dans la lignée des études réalisées dans le champ de l'ergonomie orientée enfant [DEC 15]. 
Notre objectif est d'analyser l'activité des enfants lorsqu'ils créent des histoires avec Lunii, en prenant en compte les différentes médiations, mais aussi de comprendre l'influence que peut avoir un tel dispositif sur le développement de la créativité chez l'enfant. Nous posons les questions suivantes : une interface tangible, tel Lunii, permet-elle de favoriser l'imagination? Peut-elle servir de levier aux activités narratives et créatives?

\section{Caractéristiques de Lunii - La fabrique à histoires}

Lunii prend la forme d'une petite radio et permet au sujet de composer son histoire en choisissant parmi un ensemble d'éléments répartis en quatre catégories (Tableau 1). A partir des choix réalisés par l'enfant, une histoire est générée (parmi les quarante-huit histoires pré-enregistrées). Matériellement cette interface tangible comporte une molette rotative pour faire défiler les éléments narratifs ainsi que divers boutons (menu principal, play/pause, validation), un port USB (pour recharger et télécharger des histoires supplémentaires en le connectant à un ordinateur), un port jack pour brancher un casque, une molette pour le volume qui fait également office de bouton ON/OFF. On trouve un écran sur lequel apparaissent des silhouettes lumineuses inanimées, exclusivement lors du temps de paramétrage.

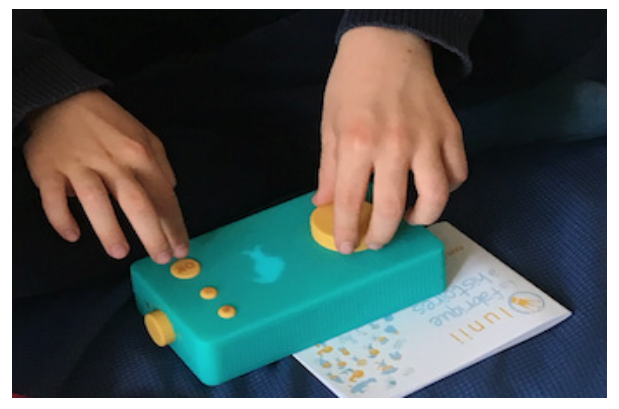

Figure 1. Lunii lors du paramétrage

\begin{tabular}{|c|c|c|c|}
\hline Personnage principal & Lieu de l'histoire & Personnage secondaire & Objet \\
\hline \multirow{24}{*}{ Suzanne ou Gaston } & \multirow{6}{*}{ Château } & \multirow{2}{*}{ Fantôme } & Balai \\
\hline & & & Bonnet \\
\hline & & \multirow{2}{*}{ Ogre } & Plume \\
\hline & & & Yaourt \\
\hline & & \multirow{2}{*}{ Princesse } & Lunette \\
\hline & & & Planche \\
\hline & \multirow{6}{*}{ Mer } & \multirow{2}{*}{ Pirate } & Robinet \\
\hline & & & Ticket \\
\hline & & \multirow{2}{*}{ Baleine } & Brosse à dents \\
\hline & & & Poivre \\
\hline & & \multirow{2}{*}{ Sirène } & Fromage \\
\hline & & & Clef \\
\hline & \multirow{6}{*}{ Forêt } & \multirow{2}{*}{ Loup } & Bonbon \\
\hline & & & Echelle \\
\hline & & \multirow{2}{*}{ Sorcière } & Lampe \\
\hline & & & Bobine de fil \\
\hline & & \multirow{2}{*}{ Lutin } & Champignon \\
\hline & & & Porte-clefs \\
\hline & \multirow{6}{*}{ Maison } & \multirow{2}{*}{ Baby-sitter } & Bille \\
\hline & & & Collier \\
\hline & & \multirow{2}{*}{ Souris } & Chaussures \\
\hline & & & Dent \\
\hline & & \multirow{2}{*}{ Père-Noël } & Araignée \\
\hline & & & Livre \\
\hline
\end{tabular}

Tableau 1. Ensemble des éléments de composition 


\section{Des objets communicants à destination des enfants}

A partir des années 2000 on assiste au développement de diverses interfaces, exclusivement numériques ou hybrides, soutenant différentes formes de narration (écrite, verbale, imagée, etc.). Certains de ces dispositifs travaillent au développement de la litéracie émergente à laquelle Lunii participe. Ce terme signifie que la lecture et l'écriture font partie d'un processus continu qui commence dès les premières années de l'enfance, les connaissances et compétences rattachées se développent avant leur apprentissage en milieu éducatif [SYL 12] [MCK 09]. Nous regardons ici trois dispositifs qui marquent une progression quant à l'espace laissé à la créativité de chaque enfant.
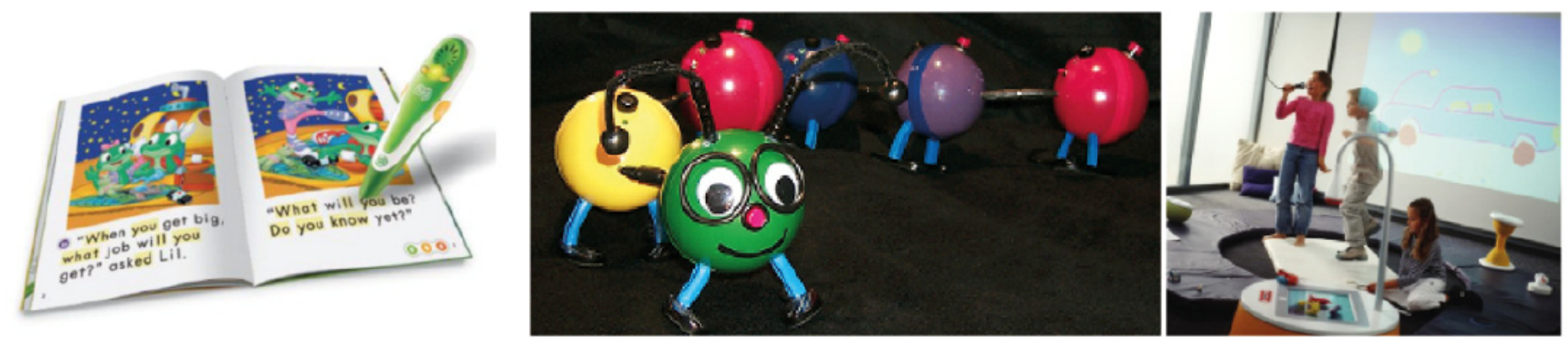

Figure 2. Tag Reading System, TellTale, POGO (de gauche à droite)

\subsection{Tag Reading System}

Le stylo lecteur Tag Reading System de Leap Frog offre une expérience connectée de la lecture à l'enfant. Il s'utilise avec un livre spécifique agrémenté de zones interactives. A l'aide du stylo, l'enfant découvre le livre en visualisant les textes et les images (pour un enfant non-lecteur) ou en le lisant, tout en activant des sons pré-programmés et émis par le stylo. Le dispositif est conçu de manière à engager l'enfant comme le parent. L'expérience de cette lecture-écoute est étendue par des jeux divers intégrant des contenus de licences connues (Disney, Nickelodeon, etc.). L'engagement de l'enfant passe aussi par la satisfaction ressentie en utilisant le Tag, les enfants ayant un vocabulaire très étendu peuvent se focaliser uniquement sur les mots inconnus, alors que d'autres encore non-lecteurs peuvent le lire en entier comme un livre classique. Les parents sont mobilisés par les données produites par l'usage du Tag, ils peuvent obtenir des informations sur la lecture de leurs enfants (temps passé sur chaque livre, jeu ou activité, niveaux complétés, nombre de réponses fournies et pourcentage de réponses correctes, etc.). Ces fonctionnalités sont possibles grâce au feedback présent dans la structure du Tag.

L'enfant, en cours d'acquisition de la lecture, va explorer des textes au delà de sa zone de confort. Il invite l'enfant à aller vers des mots inconnus, des mots qui correspondent à un niveau plus avancé de son apprentissage. Il développe alors une posture de recherche de signification et de prononciation de chaque mot, dans les livres intégrés au système Tag mais également dans les livres classiques. Cet instrument de lecture indépendante permet de cultiver le goût de la lecture tout en éveillant leur curiosité [GRA 09], éléments essentiels à la création narrative.

Le Tag a des similitudes avec Lunii, notamment quant à la proposition d'un contenu pré-établi, il ne permet pas de créer un contenu nouveau. C'est également un dispositif qui repose sur l'écoute, lui permettant d'être utilisé par des enfants non-lecteurs. Néanmoins à la différence de Lunii sa visée pédagogique est plus définie.

\subsection{TellTale}

TellTale est un outil de construction d'histoire basé sur la voix qui prend la forme d'une chenille, l'enfant y développe ses compétences narratives en enregistrant des fragments de récit. Pour ce faire il lui suffit de presser un bouton pour enregistrer un son durant vingt secondes, il peut réitérer cela avec chacun des cinq modules. Il a également la possibilité de revenir sur son enregistrement en pressant un second bouton. Sa structure repose sur un système modulaire, chaque module est détachable et peut 
être placé à l'emplacement souhaité par l'enfant. C'est uniquement lorsque le sixième module, à savoir la tête, est attaché au reste des modules que l'histoire peut être entendue.

La structuration en module tangible distinct permet, au delà d'une certaine liberté dans la composition et l'arrangement de l'histoire, un repérage de la structure narrative plus aisée pour l'enfant. Il est possible de pointer physiquement un module de la chenille, elle permet alors d' « aider les jeunes à créer et expérimenter avec la structure et le contenu du langage oral » [ANA 01]. TellTale offre à l'enfant la possibilité de créer, segmenter, organiser, lier des éléments du langage, à la différence des magnétophones Fisher Price dont son concepteur s'inspire (avec les magnétophones classiques l'enfant enregistre puis écoute, et éventuellement efface et reenregistre).

De la même manière que Lunii et le Tag, aucune dépendance à l'ordinateur ne s'installe, le dispositif forme un ensemble autonome utilisable par un enfant encore pré-lecteur étant donné que ces types d'interface reposent principalement sur un système audio. Néanmoins ici l'enfant dispose d'un contrôle du contenu comme de la structure du récit, c'est lui qui en est l'auteur-créateur. TellTale fait partie, de ce que Cassel nomme les Story Listening System (SLS) [CAS 04], distinguables selon quatre caractéristiques : l'usage de récits oraux comme amorce à la litéracie, l'encouragement du jeu entre pairs, la favorisation de dispositifs intégrés aux jeux et jouets de l'enfant évinçant ainsi les situations où il utilise des dispositifs munis d'écran-clavier-souris, et enfin le placement de l'enfant en tant que constructeur et non consommateur du récit. Lunii comporte certaines de ces caractéristiques à l'exception de la dernière étant donné que Lunii n'accueille pas de contenu extérieur.

\subsection{POGO}

$P O G O$ est un ensemble d'objets communicants permettant aux enfants de six à huit ans de créer des récits en faisant dialoguer les environnements physiques et virtuels [DEC 02]. Pour ce faire, $P O G O$ propose dix instruments, très différents de la création d'histoire sous forme d'écrit, dont : un Beamer qui permet de visualiser en temps réel et de capturer des éléments du monde physique de l'enfant puis de les importer dans l'environnement virtuel ; une caméra pour filmer des scènes et les manipuler ; un micro ; des cartes servant de mémoire mobile ; deux Mumbos qui permettent de lire et de projeter des contenus numériques ; un écran de projection horizontal ou vertical ; etc.

$P O G O$, en plus d'intégrer les outils traditionnels de construction narrative (papier-crayon, dessins, etc.), permet de créer des éléments de l'histoire, de construire des décors, d'en modifier des éléments, d'insérer des séquences vidéo, etc [DEC 15]. Cet ensemble d'artefacts disséminés se veut à l'opposé de dispositifs qui limitent la spontanéité et l'interaction avec l'environnement de l'enfant comme les CDROMs interactif de création narrative commercialisés entre la fin des années 90 et début 2000 (nous trouvons à titre d'exemple les CD-Roms de la série « Fais ton histoire ! » créée par Disney Interactive ou Adibou 2 datant de 1999). Comme analysé par les concepteurs de POGO, ces CD-ROMs de création ne permettent pas une réelle construction de récits et engendrent une perte d'intérêt rapide. L'enfant est seulement dans la sélection et la combinaison de modules, comme par exemple l'organisation de l'arrière-plan, l'ajout de sons ou d'actions pré-programmées, le choix des éléments s'avère limité tout comme les possibilités d'animation de ces éléments [DEC 02b].

En effet, à la différence de Lunii, $P O G O$ ne propose aucun contenu préétabli à l'enfant, la seule limite qu'il rencontre est celle de son imagination. La création narrative repose sur l'expérience de l'enfant [DEC 15], c'est pourquoi il était primordial que $P O G O$ propose des fonctionnalités de captation afin de créer une continuité entre l'environnement de l'enfant et l'espace du récit.

$P O G O$ représente donc un exemple de dispositif très ouvert, tant au niveau du contenu que de la forme, étant donné qu'il accueille la richesse de l'expérience dans la pluralité des modes d'expression propres à l'enfant. TellTale comme $P O G O$ permettent aux enfants de créer leur propre contenu, à la différence du Tag et de Lunii où le contenu est déjà intégré lors de l'étape de conception. POGO a aussi la singularité de convoquer une multitude de mode d'expression sans se restreindre à la voix (enregistrement et écoute) comme c'est le cas dans les différents dispositifs dont il est ici question. 


\section{Méthodologie et cadres théoriques}

Dans le cadre de notre étude sur Lunii, nous avons réalisé différentes observations en situation auprès de dix enfants. Les méthodes de recueil de données mobilisées furent l'observation avec captation audio et vidéo lors d'une séance de prêt et de retour du dispositif chez les sujets, complétés par des entretiens semi-directifs avec les enfants. Ces études sont enrichies par les travaux d'étudiants en ergonomie réalisés à la demande de F. Decortis et moi-même, permettant d'étoffer la richesse des observations. Afin d'analyser les pratiques véhiculées par cet artefact, nous mobilisons l'approche instrumentale [RAB 95] avec les médiations et genèses instrumentales, ainsi que le modèle NAM [DEC 13] pour comprendre ce qui se joue au niveau de la créativité.

\begin{tabular}{|l|l|l|}
\hline \multicolumn{1}{|c|}{ Sujets } & \multicolumn{1}{c|}{ Age des sujets } & \multicolumn{1}{c|}{ Co-activité } \\
\hline Théo & 4 ans & Accompagné d'un parent \\
\hline Paul & 10 ans & Absence de co-activité \\
\hline Sarah & 7 ans & Absence de co-activité \\
\hline Paul avec Sarah & & Frère et sœur \\
\hline Sacha & 9 ans & Absence de co-activité \\
\hline Garance & 4 ans et demi & Absence de co-activité \\
\hline Sacha avec Garance & & Frère et sœur \\
\hline Léo & 7 ans & Absence de co-activité \\
\hline Romy & 10 ans & Accompagnée d'un parent \\
\hline Amélie & 8 ans & Absence de co-activité \\
\hline Arthur & 5 ans & Accompagné d'un parent \\
\hline Amélie avec Arthur & & Frère et sœur \\
\hline
\end{tabular}

Tableau 2. Ensemble de situations observées impliquant dix enfants (pour lesquels les prénoms ont été changés par soucis d'anonymisation)

\section{L’approche instrumentale}

L'approche instrumentale, mobilisée ici pour étudier l'usage d'une interface tangible, permet d'intégrer l'usage des outils à l'analyse de l'activité sans se limiter aux buts et problèmes rencontrés par l'homme. Cette approche s'inscrit dans le champ des théories de l'activité, posant les artefacts comme des médiateurs de l'activité des sujets. Elle permet d'étudier à la fois les différentes genèses, mais aussi de prendre en compte la manière dont les instruments se constituent pour le sujet : elle vise une compréhension profonde des moyens de l'activité. On s'attache à l'usage d'une technologie en situation, en se dégageant du point de vue technocentré.

\subsection{De l'artefact à l'instrument}

Le concept d'instrument est au coeur de cette approche. Il est défini comme une entité mixte, formée de deux composants : un artefact associé à un schème d'utilisation, c'est à dire associé à un ensemble structuré des caractères généralisables de l'action qui permettent de répéter la même action. Le terme d'instrument désigne l'artefact (l'artefact étant considéré comme outil « nu ») en situation, inscrit dans un usage : il est un moyen d'action pour le sujet. On appréhende un artefact du point de vue du sujet et de son activité. Il faut retenir que l'instrument n'est pas donné mais fait l'objet d'une élaboration par le sujet, mais aussi que l'insertion de l'instrument dans l'activité comme moyen engendre des réorganisations de l'activité. 


\subsection{Des médiations variées}

Au cours de l'activité, l'instrument remplit plusieurs médiations orientées dans différentes directions : des médiations tournées vers l'objet de l'activité qualifiées d'épistémiques lorsqu'elles visent une connaissance de cet objet, ou qualifiées de pragmatiques lorsqu'il y a une action sur cet objet, des médiations interpersonnelles tournées vers les autres sujets présents ou encore des médiations réflexives lorsqu'elles sont orientées vers le sujet lui-même. Nous les mobiliserons ponctuellement pour comprendre les interactions que les enfants entretiennent avec le Lunii en prenant en compte les quatre pôles de l'activité : sujet (celui qui agit), objet de l'activité (ce que le sujet cherche à faire) pouvant être multiple et évoluant dans le temps, l'instrument ainsi que les autres sujets.

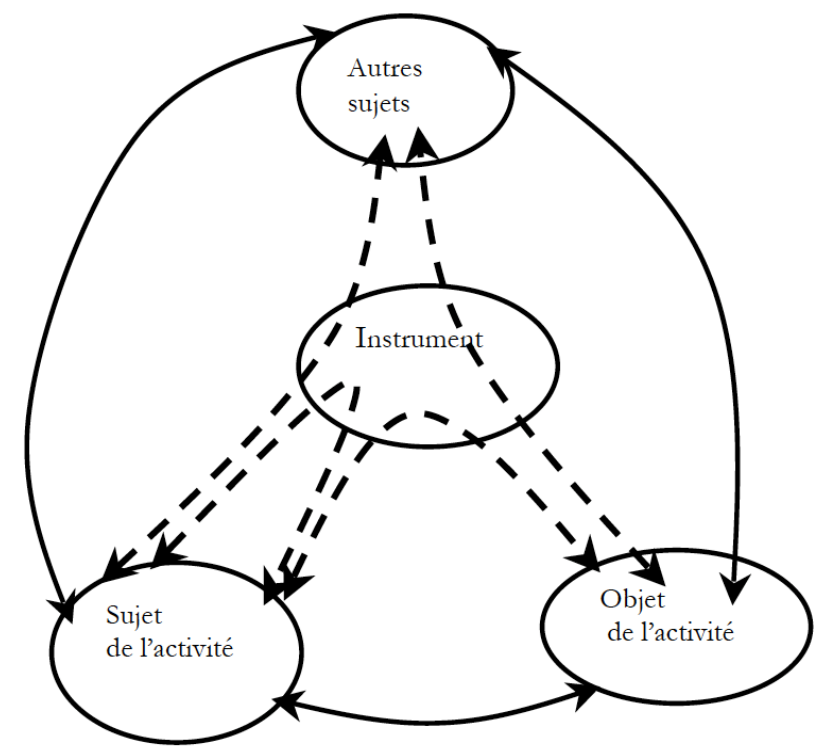

Figure 2. Modèle quadripolaire de P.Rabardel

\subsection{Genèses instrumentales}

Au cours de l'activité se mettent en place des genèses durant lesquelles le sujet cherche à construire et transformer des instruments tout en se transformant lui-même, selon un double mouvement.

\subsubsection{Processus d'instrumentation}

L'instrumentation est le premier mouvement, dirigé vers le sujet. Il correspond à l'émergence et l'évolution des schèmes : construction, évolution par accommodation, combinaison, assimilation d'artefacts à des schèmes déjà constitués [RAB 95]. Dans ce cas, c'est le sujet qui va s'adapter à l'artefact en développant de nouvelles compétences ou adapter des schèmes existants.

L'usage de Lunii se structure en deux étapes principales : le temps de paramétrage et le temps d'écoute, autour desquels plusieurs schèmes s'articulent. Nous allons ici présenter le schème d'immersion et le schème de projection-introjection. Le schème de découverte et le schème de mixage seront mis en rapport plus tard au sein du modèle NAM.

\subsubsection{Schème d'immersion}

Lorsque l'enfant s'immerge il écoute l'histoire avec toute son attention en identifiant chaque détail. L'immersion passe essentiellement par le son étant donné qu'aucune illustration n'est projetée. C'est au travers de l'intonation, du rythme et des différents bruitages que l'enfant se laisse emporter. Ce schème est assimilable sur certains points à la définition du Flow de Csikszentmihalyi [CSI 90]. Cette expérience optimale réduit les distractions et permet au sujet de se concentrer exclusivement sur ce qu'il fait, il est de ce fait totalement engagé. L'enfant plonge entièrement dans l'activité d'écoute du Lunii, oubliant les contraintes externes comme le temps et laissant place à un sentiment de bien-être comme le confirme ce verbatim de la maman de Théo : « Il s'est posé sur le lit, et il l'écoutait vraiment 
en mode attentif, vraiment posé entre les deux oreillers sur notre lit... Il adore ça, ça le canalise beaucoup, ça l'apaise ».

Des enfants manifestent des émotions, comme Théo qui avoue avoir très peur en entendant le cri de la baleine, ou qui extériorise ses souhaits : «J'ai pas envie que Gaston il nage avec la baleine, sinon la baleine elle va le manger ». Des enfants vont jusqu'à stopper l'histoire quand celle-ci ne les satisfait pas : « je ne veux pas ça, on change, on arrête ». Garance souhaitait en effet ajouter une princesse à l'histoire, malheureusement Lunii ne permet ni d'influencer le récit ni d'ajouter un personnage supplémentaire. L'immersion peut être contrariée également lorsque le sujet prête plus attention aux boutons qu'au récit. Pour Théo, parmi les quatre histoires paramétrées lors de la séance de prêt, seulement une seule a été écoutée en intégralité. Les trois autres ont été interrompues de différentes manières (bouton pause puis bouton accueil, bouton accueil, baisse du son).

\subsubsection{Schème de projection-introjection}

Le schème de projection-introjection se nourrit de celui d'immersion et comporte deux versants. Il s'agit d'un schème de nature créative : les enfants s'imaginent l'histoire dans leur tête, rattachent l'histoire à leur expérience vécue ou se mettent à la place du personnage principal.

Il y a introjection lorsque les sujets ramènent des éléments du récit à eux-mêmes. Lors d'une discussion autour de l'absence d'images sur le dispositif, Paul affirme trouver cela bien car il s'imagine l'histoire dans sa tête, Sarah verbalise ce qu'elle s'imagine : " moi j'imaginais le monsieur, et il conduisait, comme une voiture. Ensuite c'était comme des sièges, y avait la petite fille mais y avait pas de ceinture, et c'est pour ça qu'elle pouvait tomber...». On remarque que l'absence de visuels laisse un espace libre à investir pour les sujets. L'enfant intériorise certains éléments du récit, qui prennent corps dans son imaginaire.

Il y a projection lorsque les sujets projettent leur expérience vécue sur l'histoire. Des éléments narratifs font surgir l'expérience, lorsque Théo entend parler d'un bateau pneumatique il s'exclame «Sans GPS il fait n'importe quoi! ». La maman explique ensuite que le sujet fait référence à ses dernières vacances où ils avaient utilisé le GPS. L'écoute du récit stimule une forme de remémoration de l'expérience, l'enfant passe au second niveau de l'expérience [ENG 99] en prenant du recul et en réorganisant ce qu'il a vécu.

Lorsqu'il se produit une identification avec le héros, l'enfant s'imagine à sa place et en même temps accueille le personnage fictif comme s'il le remplaçait telle une identification. On retrouve cette dimension au travers du choix récurrent du personnage principal : «moi je suis un garçon alors je préfère Gaston » (Théo). L'enfant va jusqu'à se mettre dans la peau du personnage : « Moi je veux pas aller sur le dos d'une baleine ». L'enfant manifeste ouvertement son désaccord, en employant un pronom à la première personne, comme s'il était le héros.

\subsubsection{Processus d'instrumentalisation}

L'instrumentalisation représente le second mouvement dirigé cette fois vers l'artefact. Il peut être défini comme un processus d'enrichissement des propriétés de l'artefact par le sujet, c'est le sujet qui va adapter l'artefact (le personnaliser, l'enrichir des contenus nouveaux, etc.). En d'autres mots au lieu de transformer son activité, c'est le sujet qui cherche à transformer l'artefact.

Les sujets manifestent explicitement leur volonté de modifier le fonctionnement et de rendre le contenu plus personnel : « quand y a plus de choix ton histoire elle est plus à toi » (Paul). Les histoires pré-enregistrées paraissent impersonnelles dû au manque de possibilités : «On n’a pas trop le choix donc on ne peut pas dire qu'on crée l'histoire » (Sacha). La logique de la sélection dépeinte par Manovich pour les logiciels de création [MAN 10] n'est pas si éloignée du processus de paramétrage proposé par Lunii où le sujet vient piocher dans le déjà-là sans avoir la possibilité d'amener ses propres éléments. Certains revendiquent plus qu'une diversification, ils recherchent une posture d'auteur : « Je voudrais créer mes personnages [...] je pourrais décider de leur intelligence, leur corps, la personnalité 
que peut avoir mon propre personnage » (Sacha). Or l'instrumentalisation est empêchée puisque Lunii ne laisse aucune modification possible.

\section{Impacts sur l'imagination de l'enfant}

L'imagination et la créativité ont une place privilégiée dans le développement de l'enfant chez Vygotski, ils devraient être cultivés et intégrés à l'apprentissage [VYG 10]. L'imagination se base sur deux mécanismes : l'expérience et la réélaboration de l'expérience. L'expérience repose sur l'ensemble des perceptions internes et externes, ici enrichi et médiatisé par Lunii. Vient ensuite le temps de réélaboration où le sujet dissocie ses impressions, formant encore un tout complexe. Il va les séparer et n'en sélectionner que quelques unes, pour ensuite les associer. Peut-on identifier des mécanismes de l'expérience et de réélaboration de l'expérience lors de l'usage de Lunii ?

\subsection{Un modèle d'analyse de l'activité narrative et créative}

Le modèle NAM [DEC 13] reprend le cycle de Vygotski avec un grain plus fin, et s'avère intéressant à mobiliser pour appréhender une situation dans sa globalité avec une certaine systématicité. De quelle manière l'activité de l'enfant avec Lunii se développe au sein des quatre phases de NAM que sont l'exploration, l'inspiration, la production et le partage ?

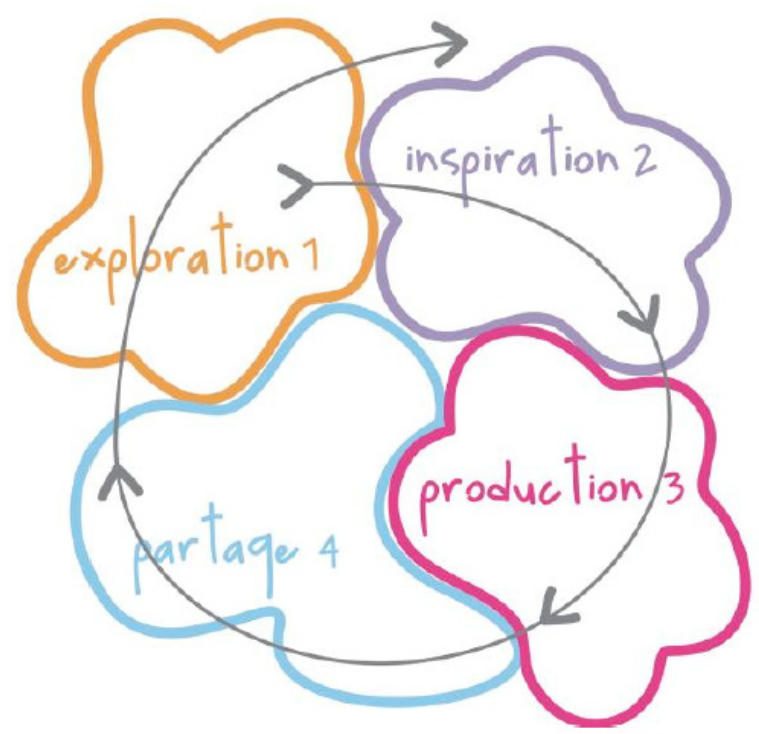

Figure 3. Modèle d'Activité Narrative (NAM « Narrative Activity Model ») de Decortis

\subsubsection{L'exploration}

Il s'agit de l'ensemble des activités qui naissent de l'expérience : ce qui a été vu, entendu, touché... Les enfants vont butiner, chercher, récolter, c'est une phase de collecte où les éléments pré-enregistrés sont encore perçus comme un tout indivisible. L'exploration se fait ici par étapes : le sujet découvre d'abord le personnage principal, puis le lieu, suivi du personnage secondaire, etc. Cette découverte est guidée par la voix pré-enregistrée du Lunii qui fait office de didacticiel («qui sera le héros de ton histoire ?", «où ton histoire va-t-elle se passer ?», etc.), elle accompagne l'enfant dans sa composition de l'histoire. Le packaging de Lunii, sur lequel figure des illustrations de tous les éléments présents (château, forêt, balai, plume, etc.), supporte également des médiations épistémiques puisqu'il rend visible le contenu du dispositif en un regard.

Le degré d'ouverture de la phase d'exploration varie en fonction des buts fixés par les sujets. Certains décident de faire toutes les histoires possibles : « je sais pas si on est arrivé à toutes les faire, parce que même aujourd'hui on en a encore trouvé » (Paul). Dans cette optique les sujets tentent de se souvenir des éléments déjà combinés pour ne pas les resélectionner : " quand on voit qu'on tombe sur la même histoire, bah on quitte » (Paul). Les sujets acquièrent une connaissance du dispositif à chaque 
nouvelle histoire réalisée, mais restreignent au fur et à mesure le champ de collecte avec un effet entonnoir.

Le degré d'ouverture de la phase d'exploration varie dans le temps notamment lorsque les sujets cherchent à reproduire les histoires appréciées. Théo a exploré sans a priori lors des premières utilisations, puis une fois ses préférences trouvées, l'exploration c'est interrompue. Elle a été relancée par l'intervention du parent : «Je me suis amusée à en choisir une parce que sinon il écoute toujours les mêmes [...] Du coup j'en ai fait une autre et il a bien aimé. Là après il a changé la sienne et il en a créé une autre ». Sans l'intervention du parent le sujet aurait probablement continué à survoler la phase d'exploration pour aller vers des choix quasi-automatiques.

\subsubsection{Schème de découverte}

Un premier schème s'organise autour de la découverte des éléments narratifs, que nous avons appelé schème de découverte. L'enfant fait défiler les différents contenus dont il ignore l'étendu, il chemine dans un univers narratif. L'attention de l'enfant est partagée entre écouter le Lunii, parfois le réécouter, et regarder les illustrations lumineuses qui s'affichent. La découverte passe par la molette rotative ainsi que par le langage lors de co-activité. Le sujet est face à des éléments inconnus qu'il interprète en fonction de son vécu comme Garance qui choisit le géant puisqu'il lui rappelle un personnage d'un film d'animation qu'elle apprécie.

\subsubsection{L'inspiration}

L'inspiration, c'est le moment où se fait le choix des idées à développer, on donne de l'importance à certains éléments. Il s'agit d'une pratique réflexive où les enfants dissocient ces touts complexes constitués. Conformément au modèle NAM initialement établi, la réflexion est individuelle voire introspective, or ici avec Lunii la réflexion peut se mêler au partage et faire l'objet de débat. L'inspiration suit ici le rythme progressif de l'exploration, elle est scandée par un choix obligatoire pour progresser dans le paramétrage de l'histoire.

L'inspiration s'avère extrêmement dépendante du temps d'exploration car c'est cette dernière qui conditionne le choix à un nombre d'éléments pré-définis et pré-enregistrés. Cette restriction peut conduire des sujets à effectuer des choix par défaut comme ici : " Bon bah une brosse à dent alors » (Sacha). Cette limitation aux éléments proposés par Lunii peut faciliter le choix, mais aussi provoquer de la frustration chez les sujets, les conduisant pour certains à interrompre l'usage du dispositif.

Au contraire certains sujets semblent effectuer un choix délibéré notamment lorsqu'ils se fixent euxmêmes d'autres objets de l'activité : « Sarah elle voulait faire toutes les histoires où y avait des souris » (Paul). Ici le sujet se fixe une règle et restreint par lui-même le champ de possibles offert par le dispositif en orientant sa recherche. Dans ce cas particulier, la phase d'inspiration surplombe totalement la phase d'exploration en la faisant disparaître (notons tout de même que cette démarche advient suite à une phase d'exploration antérieure qui permet d'orienter en amont le champ de collecte à tous les éléments en lien avec le personnage de la souris). Dans ce type de situation, le déplacement de l'objet de l'activité permet le développement de médiations pragmatiques, on observe le glissement de l'objet d'origine de l'activité (« créer et écouter une histoire ») vers des objets comme « reproduire une histoire précédente » (Théo) ou « réaliser toutes les histoires possibles » (Paul), qui restreignent ou ouvrent l'ensemble des choix possibles.

\subsubsection{Schème de mixage}

En complément, nous trouvons un schème de mixage. Les enfants réfléchissent, puis appuyent sur le bouton OK pour choisir, parfois en mobilisant le langage lors de co-activité. Ils comprennent que le choix des éléments permet d'avancer dans le paramétrage du récit. Les sujets paramètrent leurs récits en faisant cohabiter le schème de découverte avec le schème de mixage, qui peuvent s'enchainer de manière linéaire tout comme se rencontrer lors d'allers-retours entre ces deux schèmes. Nous pouvons utiliser la métaphore de la préparation d'une recette de cuisine où l'on sélectionne des ingrédients à associer ensemble. 


\subsubsection{La production}

La production, pendant cette phase a lieu l'association des éléments dissociés et l'expression. On exploite les éléments choisis lors de la phase précédente, on les retravaille pour produire de nouveaux contenus : on est dans une extériorisation qui vise à prendre en compte les différents moyens d'expression mais aussi les éventuels lecteurs ou auditeurs qui recevront l'histoire.

On assiste à la production d'éléments narratifs durant l'écoute du Lunii. Théo répète des éléments et les complète avec son avis personnel : "C'est Clémentine comme Valentine », "Les pirates c'est méchant », «Les monstres c'est pas bon ». Le sujet en question ne se limite pas à la répétition, il introduit des éléments extérieurs au récit par exemple lorsqu'il associe le personnage de Clémentine à celui de Valentine (Valentine est le prénom d'une petite fille qu'il voit quotidiennement). Le rattachement à son expérience personnelle passe par l'histoire racontée par Lunii mais aussi par les illustrations lumineuses qui s'affichent lors du paramétrage, lorsque Lunii énonce le lieu «Une chambre », la silhouette d'un lit apparait, Théo s'exclame « Moi j'ai un grand lit, là il est tout petit. Et dessous de mon lit $\mathrm{y}$ a un tout petit lit». Nous relevons qu'il s'agit des seules productions synchroniques observées.

D'autres productions, variées et diachroniques, adviennent si l'on prend en compte l'activité une fois le Lunii éteint. L'éventail des productions est très diversifié : Romy a réalisé un dessin de baleine ; F4 après avoir parcouru le contenu du Lunii et sélectionné Suzanne comme personnage central, délaisse le Lunii pour aller jouer avec ses poupées en en prénommant une Suzanne ; Léo a inventé et rédigé une histoire reprenant des thèmes présents dans le Lunii quelques jours après l'avoir écouté ; Ou encore un couple d'enfant (Paul avec Sarah) s'est engagé dans une activité autour du jeu StoryCubes en reprenant un élément entendu dans l'histoire quelques minutes avant. Ces cas de production narrative montrent le réinvestissement de Lunii par les enfants : leur imagination prend source durant l'écoute puis s'incarne une fois son usage terminé.

\subsubsection{Le partage}

Le partage, c'est le moment où l'histoire est racontée par un enfant et partagée avec autrui (camarades, amis, parents) en produisant un effet sur ces derniers. Lors de l'utilisation du Lunii nous relevons un ensemble de médiations interpersonnelles au sein de situations de partage de différentes natures :

Le partage peut être tourné vers l'apprentissage et la transmission de connaissances. Sacha, connaissant le Lunii, accompagne Garance pas à pas dans sa première utilisation du dispositif : il lui demande quels sont ses choix, répète les phrases dites par le Lunii puis les valide. Cette situation de partage illustre la Zone Proximale de Développement [VYG 34], cet écart entre le niveau de développement actuel et le niveau de développement potentiel qu'un parent ou qu'un sujet plus expert permet d'atteindre. Nous y observons des médiations interpersonnelles épistémiques puisque c'est par l'intermédiaire d'un tiers que le sujet développe des connaissances supplémentaires sur le dispositif. Ce type de partage intervient au cours de l'activité sur le Lunii principalement lors des phases d'exploration et d'inspiration.

L'aide d'un parent peut aussi advenir sur la durée totale de l'activité et prendre la forme d'une régulation. Par exemple, la maman de Théo canalise ces gestes («n’appuie pas sur les boutons ») afin que l'enfant reste ciblé sur l'objet principal de l'activité qui est l'écoute d'histoires. Ce type de partage est alors inhérent à toutes les phases de NAM dans ce cas.

Ces interactions sociales génèrent également des moments d'être ensemble parfois simplement tournés vers la découverte à autrui. Garance, enthousiasmée, fait écouter une histoire à ses petits frères, ou encore Théo raconte lui-même l'histoire qu'il a écouté seul à sa maman. Ces temps se produisent une fois l'usage de Lunii terminé. Pour le sujet il s'agit d'une découverte dont la finalité est de faire partager ce qui a été entendu, il n’y a pas vocation à apprendre à faire faire ou à faire mieux. 


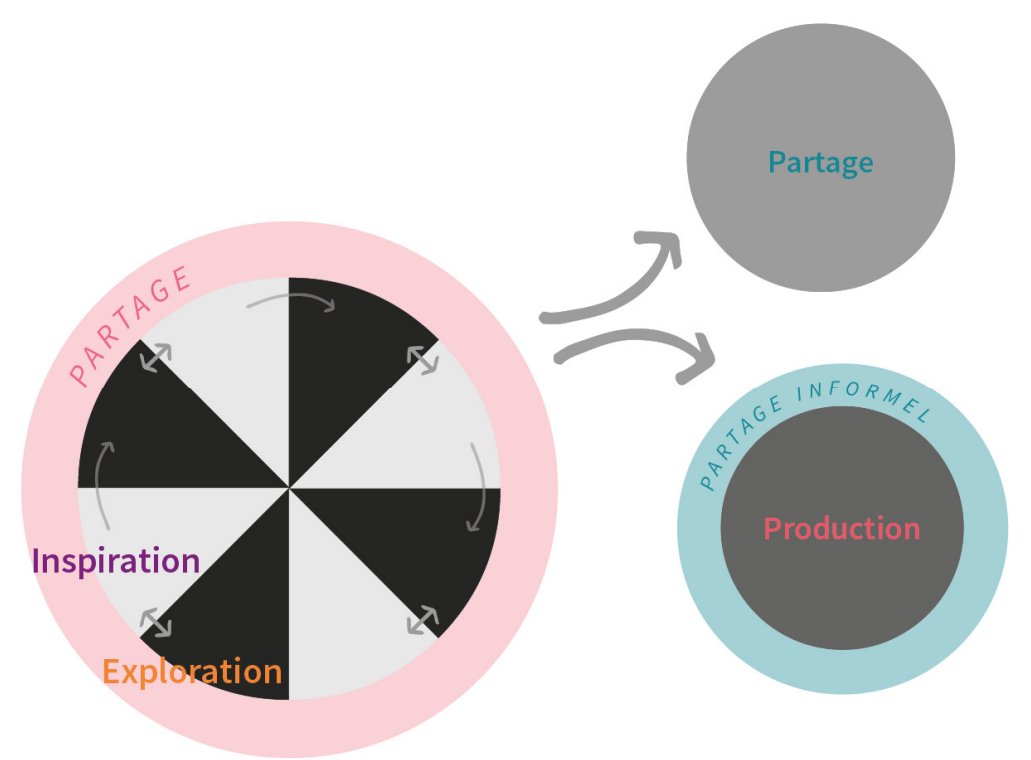

Figure 4. Modèle NAM appliqué à l'activité des enfants avec le Lunii

\section{Discussion}

Lunii pourrait alors être posé comme un instrument au service de la créativité qui offre un développement de ressources créatives et narratives, au sens où il crée des liens avec ce qui a été vécu auparavant et invite l'enfant à produire des récits variés, même diachroniques. Au cours de ces diverses productions l'enfant développe des compétences narratives tout en se transformant lui-même, ce qui nous permet de relever une prédominance des médiations réflexives, voire interpersonnelles réflexives comme pointé par Decortis dans des cas de co-activité [DEC 13].

Néanmoins on ne peut passer outre les limites pointées par les différents sujets, l'activité créative reste limitée, au moment du paramétrage (avec son contenu prédéfini non modifiable) comme au moment de l'écoute (impossibilité d'intervenir sur l'histoire). Il serait intéressant de réaliser une étude de l'usage de Lunii, d'une part sur une durée plus longue nous permettant de voir ce qu'il advient une fois l'effet de nouveauté dissipé, et d'autre part avec un nombre de sujets plus important pour confirmer les résultats généralisés.

Les objets communicants à destination d'un public enfant représentent un type d'interface pertinent à mobiliser au sein de situations d'apprentissage, étant donné qu'ils offrent des interactions plus sensibles et permettent aux enfants de manipuler des éléments auparavant abstraits. Les Tangible User Interface (TUI), pour reprendre le vocabulaire de Ishii, ne sont pas séparées des modes d'interaction que nous convoquons dans notre environnement quotidien et permettent de convoquer certaines de nos aptitudes. Elles rendent « l'information digitale manipulable avec nos mains, et perceptible par nos sens » [ISH 06]. Ce dernier point est loin d'être négligeable si l'on suit la pensée du designer-artistepédagogue Munari pour qui l'usage de tous les organes est complémentaire : « Le toucher complète la sensation visuelle et auditive, il fournit d'autres informations utiles à la connaissance de tout ce qui nous entoure » [MUN 11]. Les objets communicants permettent de combiner les vertus des interfaces exclusivement numériques avec celles des interfaces tangibles. D'une part nous ne sommes plus limités aux écrans plats et à une interaction du bout des doigts, et d'autre part le digital offre une opportunité face au manque de malléabilité d'un objet physique (il est en effet difficile de modifier la forme ou les propriétés d'un objet physique comme sa couleur ou ses dimensions).

\section{Quelques perspectives d'enrichissement}

Regardons tout de même quelques pistes d'amélioration de Lunii aiguillées par des éléments de l'activité : 
Dans des situations de co-activité, par exemple un frère avec une sœur, intégrer un mode aléatoire permettrait de régler le problème de conflit lié au moment du paramétrage.

De jeunes sujets ressentent de la peur lié au contenu des récits, des sujets plus âgés s'ennuient comme Paul en témoigne : " dans une histoire ils vont chez papi et mamie et là ils mettent la radio et là c'est Une souris verte..., c'est un peu pour petit ». En proposant différents niveaux liés à l'âge des enfants Lunii gagnerait en adaptabilité.

Des préférences se dessinent au fur et à mesure de la découverte des récits, le fait de pouvoir archiver les histoires déjà effectuées, telle une bibliothèque, permettrait aux sujets de retrouver une de leur histoire favorite rapidement. Nous éviterions des situations telles que Théo, après avoir interrompu l'histoire par mégarde, tente de refaire la même histoire mais s'érige de ne pas avoir accès à tous les contenus : «Ah le fantôme, pourquoi y a plus le fantôme ? » (Théo). La logique de paramétrage en arborescence n'est pas aisée pour les enfants souhaitant retrouver un élément particulier.

A contrario pour des sujets souhaitant paramétrer tous les histoires réalisables avec le Lunii, les éléments encore non combinés ensemble pourrait s'éclairer d'une autre couleur pour guider les enfants dans leur exploration afin d'éviter de ressentir une forme d'agacement lorsqu'il paramètre une histoire déjà réalisée alors qu'ils en souhaitaient une nouvelle.

Il serait aussi intéressant de pouvoir modifier le récit au cours de l'écoute en s'inspirant des histoires à embranchement comme les Livres Dont Vous Etes Le Héros : influencer la tournure de l'histoire en ajoutant des personnages supplémentaires (comme Garance souhaitant ajouter une princesse), ou en décidant d'une fin idyllique ou tragique par exemple.

Certains sujets (Paul, Sacha) se plaignent du panel de choix limité et formulent une envie de rendre leur histoire plus personnelle, il serait alors intéressant de permettre aux sujets de créer leurs propres contenus (personnages, lieux, objets). Cet aspect fait écho à la fertilité de l'environnement chère à Papert qui perçoit les enfants comme des « bâtisseurs », des « bricoleurs » ou encore des « architectes » [PAP 99]. La part active des enfants serait alors augmentée. Dans une perspective éducative (la lecture comme la compréhension d'un récit font partie des connaissances à acquérir en milieu scolaire), cette amélioration de Lunii permettrait de diversifier les points de contact entre la vie quotidienne de l'enfant et les compétences ou connaissances visées, tout en rendant possible un apprentissage syntone (à entendre au sens d'un apprentissage qui permet à l'enfant de trouver du sens). La création de contenus ne devrait pas être nécessairement totale, il y aurait probablement un équilibre à trouver entre les éléments pré-conçus et proposés par les concepteurs et ceux élaborés par l'enfant comme des dispositifs comme TOK - Touch, Organize, Create [SYL 15]. TOK propose une plateforme connectée à l'ordinateur agrémentée de 23 blocs représentant des décors, éléments naturels, personnages et objets extraits de récits collectif. A partir de la combinaison de ces entités l'enfant enregistre son récit dans un microphone. Les récits produits avec $T O K$ ne peuvent être pré-définis étant donné que, d'une part, des règles sont attribuées à chaque élément, et d'autre part leur comportement dépend des autres éléments placés sur la plateforme, par exemple si la princesse est attaquée par le loup l'enfant peut choisir la manière d'aider la princesse en plaçant une maison pour qu'elle s'y cache ou en intégrant un autre personnage pour l'aider.

Nous pouvons résumer ces quelques recomandations par la nécessité pour Lunii de gagner en ouverture et laisser des vides à investir comme le font, à l'extrême des dispositifs comme $P O G O$ ou dans un entre-deux des dispositifs comme $T O K$, qui placent réellement l'enfant en posture d'auteur et non en posture de simple compositeur [DEC 02].

\section{Remerciements}

Nous tenons ici à remercier les étudiants du master 1 «Ergonomie, travail, formation, vie quotidienne » de Paris 8 (T. Caquel, A. Fossier, S. Foulon Krief, C. Julien, C. Machado, E. Perrin, C. Rondepierre, P. Swartz, E. Tastayre) pour la richesse et précision de leurs travaux réalisés au sein du 
cours « Ergonomie de l'enfant et de l'adolescent», qui viennent enrichir les données récueillies. Nous souhaitons également remercier les parents et enfants qui ont accepté de recevoir Lunii, de jouer avec durant plusieurs semaines et d'échanger autour de leurs pratiques, sans eux cette étude n'aurait pu être possible.

\section{Bibliographie}

[ANA 01] ANANNY Michael J., Telling Tales : A new way to encourage written literacy through oral language, sous la direction de Hiroshi Ishii, Master of Science in Media Arts and Sciences at the MIT, Cambridge, 2001.

[CAS 04] CASSELL J., « Towards a Model of Technology and Literacy Development: Story Listening Systems », Journal of Applied Developmental Psychology 25 (1), P. 75-105, 2004.

[CSI 90] Csikszentminalyi M., Flow: The Psychology of Optimal Experience, Harper and Row, New York, 1990.

[DEC 15] DECORTIS F., L'ergonomie orientée enfant. Concevoir pour le développement, Presses Universitaires de France, Paris, 2015.

[DEC 13] DECORTIS F., « L'activité narrative dans ses dimensions multi instrumentée et créative en situation pédagogique », Activités 10-1, p. 3-30, 2013.

[DEC 02] Decortis F., Marti P., Moderini C., Rizzo A., Rutgers J., \& Thursfield P., « Usages et conception d'instruments actifs pour la créativité narrative », Les Cahiers du numérique, n4, p. 127-148, 2002.

[DEC 02b] Decortis F. \& Rizzo A., «New Active Tools for Supporting Narrative Structures », Personal and Ubiquitous Computing Volume 6 Issue 5-6, p. 416-429, 2002.

[ENG 99] ENGEL S., The stories children tell. Making sense of the narratives of childhood, W.H. Freeman, New York, 1999.

[FOL 04] FOLCHER V. \& RABARDEL P., « Hommes, artefacts, activités: perspective instrumentale », dans P.FALZON, Ergonomie, Presses Universitaires de France, Paris, 2004.

[GRA 09] Gray J., Bulat J., Cunningham A., \& JaYNeS C., «LeapFrog learning design: Playful approaches to literacy, from LeapPad to the Tag reading system » dans A. DRUIN (dir.), Mobile Technology for Children: Designing for Interaction and Learning, MA: Morgan Kaufmann, Burlington, 2009.

[ISH 06] IsHII H., « Tangible User Interfaces », Proceedings of the SIGCHI conference on Human Factors in Computing Systems, Montreal, Canada, 2006.

[MAN 10] MANOVICH L., Le langage des nouveaux médias [2001], trad. de l'anglais par Richard Crevier, Dijon, Les Presses du réel, 2010.

[MCK 09] MC KenNey S. \& VoogT J., « Designing technology for emergent literacy: The PictoPal initiative », Computers \& Education 52, p. 719-729, 2009.

[MUN 11] MUNARI B., Les ateliers tactiles [1985], trad. de l'italien par Annie Mirabel \& Agnès Levecque, Les Trois Ourses, 2011.

[PAP 99] PAPERT S., Jaillissement de l'esprit: ordinateurs et apprentissage [1981], trad. de l'anglais par Rose-Marie Vassallo-Villaneau, Flammarion, Paris, 1999.

[RAB 95] RABARDEL P., Les hommes et les technologies, approche cognitive des instruments contemporains, Armand Colin, Paris, 1995.

[SUC 87] SUCHMAN L.A., Plans and situated actions: the problem of human-machine communication, Cambridge University Press, Cambridge, 1987.

[SYL 12] Sylla C., Goncalves S., Branco P., \& Coutinho C., «t-words: Playing with Sounds and Creating Narratives » dans A. NiJHOLt, T. ROMAO, D. REIDSMA (dir.), Advances in Computer Entertainment: 9th International Conference, Springer, Berlin, 2012.

[SYL 15] Sylla C., Coutinho C., BRANCO P., \& MulleR W., «Investigating the use of digital manipulatives for storytelling in pre-school », International Journal of Child-Computer Interaction 6, p. 39-48, 2015.

[VYG 34] VyGOTSKY L.S., Pensée et langage, Editions sociales, Paris, 1934.

[VYG 10] VYGOSTKY L.S., Immaginazione e creatività nell'età infantile, Editori Riuniti, Rome, 2010. 